\title{
Karsten Igel, Thomas Lau (dir.), Die Stadt im Raum. Vorstellungen, Entwürfe und Gestaltungen im vormodernen Europa
}

\section{Michel Pauly}

\section{OpenEdition}

\section{Journals}

Édition électronique

URL : http://journals.openedition.org/ifha/8811

DOI : 10.4000/ifha. 8811

ISSN : 2198-8943

\section{Éditeur}

IFRA - Institut franco-allemand (sciences historiques et sociales)

\section{Référence électronique}

Michel Pauly, « Karsten Igel, Thomas Lau (dir.), Die Stadt im Raum. Vorstellungen, Entwürfe und Gestaltungen im vormodernen Europa », Revue de l'IFHA [En ligne], Date de recension, mis en ligne le 02 novembre 2017, consulté le 24 septembre 2020. URL : http://journals.openedition.org/ifha/8811 DOI : https://doi.org/10.4000/ifha.8811

Ce document a été généré automatiquement le 24 septembre 2020.

(C)IFHA 


\section{Karsten Igel, Thomas Lau (dir.), Die Stadt im Raum. Vorstellungen, Entwürfe und Gestaltungen im vormodernen Europa}

Michel Pauly

\section{RÉFÉRENCE}

Karsten Igel, Thomas Lau (dir.), Die Stadt im Raum. Vorstellungen, Entwürfe und

Gestaltungen im vormodernen Europa, Städteforschung, 89, Köln/Weimar/Wien: Böhlau Verlag, 2016, 361 p, $45 €$ 
Le spatial turn n'est plus très nouveau en science historique et pourtant il continue à faire l'objet de monographies, de colloques et d'ouvrages collectifs. Depuis Henri Lefebvre, l'espace n'est plus considéré comme donné, comme conteneur, mais comme production, comme construction sociale. Les éditeurs de ce volume ont demandé à 19 auteurs, spécialistes du Moyen Âge ou de l'époque moderne, historiens ou historiens de l'art, traitant d'exemples allemands, suisses et italiens, d'observer la multiplicité des espaces que génère la ville, dans un processus complexe de construction exogène et allogène qui attribue à une agglomération la qualité urbaine. Ces espaces vont de la maison et de la rue jusqu'à la ville entière et au-delà à un

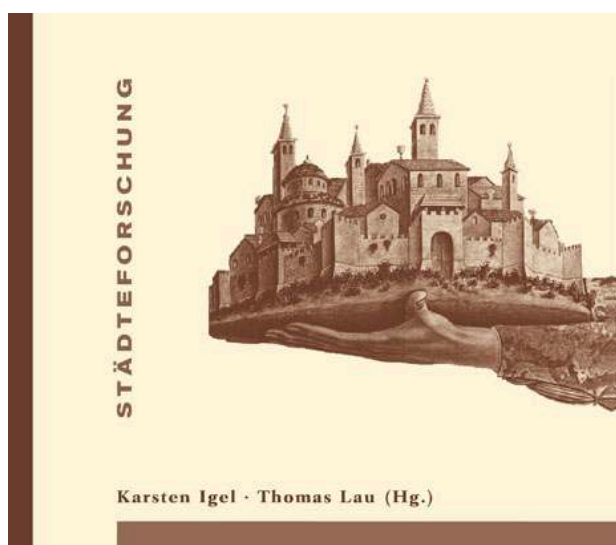

DIE STADT IM RAUM

Vorstellungen, Entwürfe und

Gestaltungen im vormodernen Europa espace supra-urbain, dont la perception peut à chaque niveau différer d'un percepteur à l'autre. La ville est alors la synthèse de ces perceptions, parfois fixée sous forme de texte ou d'image.

Les vingt contributions du volume, toutes en allemand, sont au premier abord assez disparates et n'intègrent pas toutes l'approche constructiviste de l'espace à laquelle se réfèrent les éditeurs. Nous essaierons de montrer au moins la multiplicité des niveaux d'espaces abordés. Pour ouvrir le volume, à la suite de l'introduction conceptuelle de Thomas Lau, Peter Stephan réinterprète le Jugement dernier de Michel-Ange en insistant sur sa localisation derrière l'autel principal de la Sixtine, de sorte que l'église, $\mathrm{y}$ compris le chœur et l'autel où doivent se produire le sacrifice apportant la rédemption, et partant la ville de Rome, ne font pas partie de l'espace céleste qui se dresse au-delà. En outre, les clefs du paradis détenues par Pierre sont cassées (image fréquente chez les luthériens), ce qu'aucun commentateur n'avait encore relevé jusqu'ici. Pour P. Stephan, la clef de la fresque est la femme blottie derrière JeanBaptiste que Pierre montre du doigt et que le Christ fixe de son regard : une allégorie de l'Église pécheresse. La localisation et la conception de cette scène accusatrice d'une profonde signification théologique ne sauraient être inspirées que par le pape Paul III en personne.

Vera Isaiasz continue l'interprétation d'espaces ecclésiastiques en montrant comment à Berlin, entre 1680 et 1720, la construction d'églises était source de conflits entre d'une part le souverain qui tâchait de gommer les différends entre luthériens et réformés en imposant l'utilisation des mêmes édifices par les deux confessions, et d'autre part les communautés confessionnelles et de quartiers qui devaient s'approprier ces édifices publics. Hans-Jochen Schmid analyse l'appropriation symbolique, parfois très précise, de Jérusalem et du Saint-Sépulcre à travers l'Écriture sainte et des récits de pèlerins, opposée à l'impossibilité matérielle de reconquérir la ville sainte. Sabine Reichert montre que l'imitation de Rome dans la ville de Trèves n'avait pas seulement un caractère urbanistique et architectural, mais était aussi liturgique: à travers des 
processions, la communauté s'appropriait et sacralisait l'espace urbain. Volker Reinhardt montre d'une part la transformation d'espaces religieux en espaces privés, dans le cadre du népotisme pontifical au XVII ${ }^{e}$ siècle, et d'autre part la constitution d'espaces mémoriels familiaux concurrentiels dans leur lutte contre la déchéance sociale.

Michael Hecht se pose la question de savoir si, dans l'Allemagne du nord du XVI ${ }^{\mathrm{e}}$ siècle, patriciat urbain et noblesse campagnarde, qui utilisent souvent les mêmes espaces de communication et d'action et qui poursuivent les mêmes pratiques culturelles et mémorielles, ne constituent pas plutôt des catégories d'analyse que des formations sociales clairement définies. Au XVII ${ }^{\mathrm{e}}$ siècle cependant intervient un cloisonnement de plus en plus étanche entre les états que Rita Binz-Wohlhauser analyse pour le XVII siècle à Fribourg (en Suisse), où les distinctions sociales l'emportent même au sein de l'élite urbaine, entraînant des polarisations conflictuelles; mais l'auteure ne fait guère ressortir l'aspect spatial de cette fragmentation sociale. Éric Pitz réfléchit au rôle des quartiers, espaces réduits à haute interaction sociale, et du civisme actif des habitants dans la poursuite du bonum commune dans les villes de l'époque moderne; dans un tableau récapitulatif, il envisage aussi les fonctions des paroisses, qu'il n'aborde cependant pas dans son analyse.

Au Moyen Âge, Bamberg en Bavière était composée de plusieurs entités politiques et d'espaces judiciaires, mais constituait un seul espace économique et de défense. Claudia Esch, dans une des contributions les plus intéressantes du volume, pose à juste titre la question de savoir qui construisait la ville : les différents seigneurs immunitaires ou les bourgeois? Ces conceptions divergentes de ce qu'était la ville allaient conduire au XV $\mathrm{XV}^{\mathrm{e}}$ siècle à bien des conflits. Silke Kurth prend pour point de départ les plans de Florence $\mathrm{du} \mathrm{XV}^{\mathrm{e}}$ siècle pour voir comment y sont représentées les transformations du miluogo, le milieu, c'est-à-dire cet amalgame spatio-social et culturel, notamment autour de la place du vieux marché.

Daniel Leis accède au niveau supra-urbain en identifiant dans les villes de la Terraferma les éléments architecturaux qui rappellent aux habitants qu'ils dépendent de Venise et qui constituent de la sorte ces villes vénitiennes en réseau. Emanuel Leugger analyse les relations des petites villes de Romont et de Gruyère avec leur capitale Fribourg au XVIII siècle: alors que l'élite bourgeoise de Romont, tout en défendant ses prérogatives à l'intérieur de la ville, se soumettait fidèlement à la république de Fribourg, à Gruyère les conflits entre dignitaires locaux et l'absence de fonctions centrales judiciaires facilitaient l'exercice du pouvoir par le bailli de Fribourg. Ici encore, on se demande en quoi le spatial turn a renouvelé l'analyse historique. Par contre Heinrich Speich profite de la redécouverte de la perception de l'espace pour rendre aux autres acteurs d'un territoire urbain, dans ce cas celui de Berne, leur autonomie d'action dans la négociation de conventions de protection (Burgrechte) ou de bourgeoisie, surtout avec des nobles de la périphérie urbaine. Il parvient à montrer que la constitution d'un espace juridique par les Burgrechte entraînait aussi des relations économiques et fiscales, mais n'excluait pas des conflits. Il met en question la conception de la formation territoriale au seul bénéfice de la ville centrale. Karsten Igel relate les conflits entre les bourgeois d'Osnabruck et leur évêque, seigneur de la ville, au $\mathrm{XV}^{\mathrm{e}}$ siècle. Il en conclut que le conseil urbain œuvrait certes pour préserver son autonomie, mais en maintenant l'intégration de la ville dans le territoire épiscopal, seule garantie de sa participation à l'assemblée des états du diocèse et donc à la 
politique territoriale épiscopale, sans parler des opportunités économiques et de la protection seigneuriale ainsi acquise.

Klara Hübner adhère à une conception tout à fait classique de l'analyse spatiale en insistant sur l'importance de la transmission des informations dans le territoire contrôlé et sur la rapidité de leur diffusion, et donc sur le rôle des messagers comme vecteurs du pouvoir. Bastian Walter-Bogedain, négligeant tout aspect spatial, soulève la difficulté de distinguer contacts officiels, officieux, informels et privés entre représentants d'une ville ou d'intérêts particuliers et souligne le rôle de la taverne comme lieu d'échanges. Les éditeurs auraient pu placer à la suite de cet article celui de Thomas Lau qui prend le cas des ambassadeurs des fédérés suisses engagés à côté du roi de France dans le conflit bourguignon pour se demander s'ils représentaient les intérêts de la Confédération, de certaines villes ou de certaines élites.

Marco Tomaszewski a bien intégré l'approche constructiviste de l'espace pour voir si la ville de Bâle concevait la Confédération helvétique dès 1501, année de son adhésion, comme un espace avec lequel elle pouvait s'identifier. Daniela Hacke attire l'attention sur les espaces sonores constitués par des groupements en révolte lors de la Guerre des paysans pour construire des espaces de domination, certes aux frontières peu précises, voire mobiles.

Le plus étonnant dans cette publication consacrée aux espaces urbains de différents niveaux est l'absence quasiment générale de plans, historiques ou actuels, des villes et de leur périphérie plus ou moins étendue.

\section{INDEX}

Index chronologique : Moyen Âge, Période moderne

Thèmes : Histoire des villes et des régions, Histoire sociale

\section{AUTEURS}

\section{MICHEL PAULY}

Université du Luxembourg 